\title{
Pesticides in Surface Water in the Bighorn and North Platte River Basins, Wyoming, 2006
}

\author{
By Cheryl A. Eddy-Miller, Gregory K. Boughton, and R.E. Woodruff
}

\section{Introduction}

In 2006, the U.S. Geological Survey (USGS), in cooperation with the Wyoming Department of Agriculture, sampled five surface-water sites in Wyoming - three in the Bighorn River Basin (BRB) and two in the North Platte River Basin (NPRB) (fig. 1). The purpose of the sampling was to describe the occurrence of pesticides in these basins during three different times of the year. This fact sheet presents the results of the sampling.

\section{Approach}

Three samples were collected from each site, one each in March, May, and September 2006 (table 1; fig. 2). The sampling events corresponded with pre-application of pesticides, post application of pesticides, and late growing season. The samples were collected using standard methods described in the National Field Manual for the Collection of Water-Quality Data (U.S. Geological Survey, 1997 to 2004) and analyzed at the USGS National Water Quality Laboratory, to determine the presence and concentration of 132 different pesticides and degradation products (table 2) (Furlong and others, 2001, and Sandstrom and others, 2001).

\section{Site Descriptions}

Four of the five selected sites were located at the farthest downstream site available on the river in order to integrate drainage from as much land use in the river basin as possible. The fifth site is located on the mainstem of the Bighorn River at Basin (station number 06274300). This additional site on the Bighorn River was selected because of the large drainage area of the river.

All sampling locations in the BRB were located at sites with active streamflow-gaging stations. The sampling site near Torrington (station number 06674500) in the NPRB was located at an active gaging station. The Laramie River sampling site near Wheatland (station number 06664000), is a historic gaging station, but was not active at the time of sampling. This sampling site is the farthest downstream site on the Laramie River with an active or inactive gaging station, that is upstream from Grayrocks Reservoir.

\section{Results}

Samples collected in both the BRB and NPRB contained detectable concentrations of pesticides for all three sampling events. Eighteen different pesticides and degradation products were detected (table 1).

Sixteen different pesticides were detected in water samples collected in the BRB. The most commonly detected pesticide was atrazine, a selective herbicide commonly applied to corn, grains, and sorghum (Meister, 2002), detected at low concentrations in all nine samples collected in the BRB. The pesticide with the largest concentration in the BRB was the herbicide MCPA (trade names 


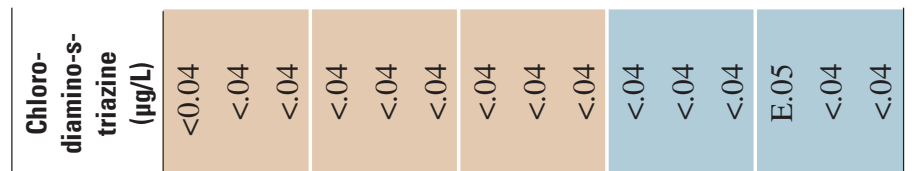

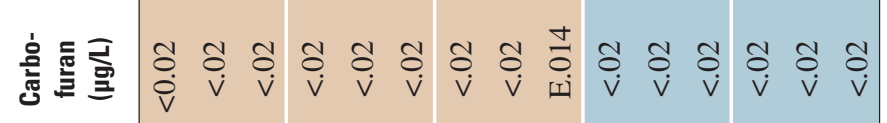

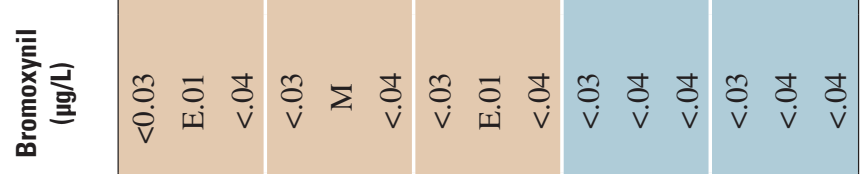

安

西

茑

营

뭏

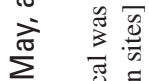

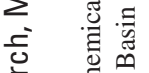

का

贾

离

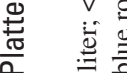

E

$\sum^{\circ}$

ल

言

兽 唯

焉

音

若

弯

离

害

$\sum_{i}^{\infty}$

E

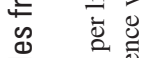

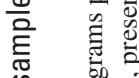

$\stackrel{5}{=}$

들

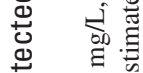

峞

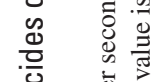

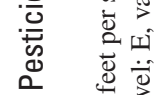

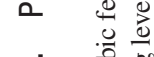

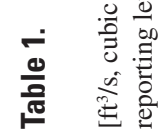

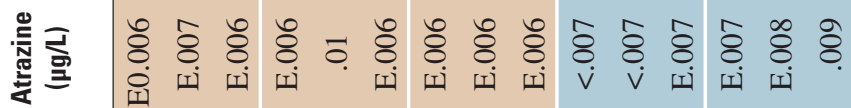

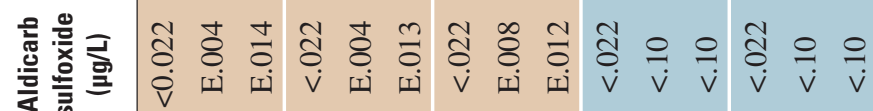

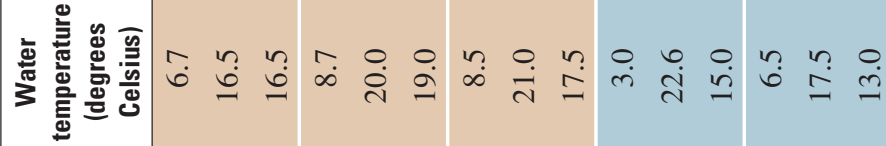

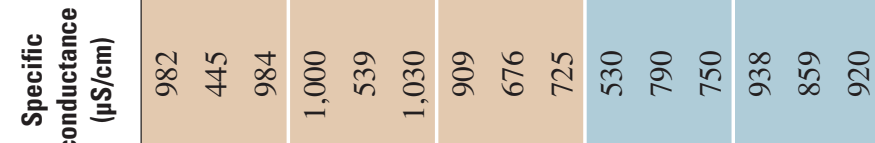

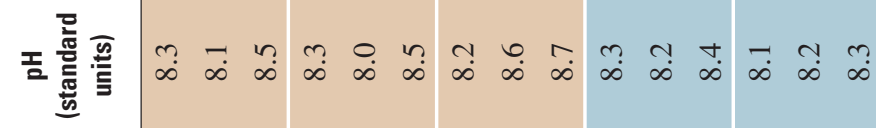

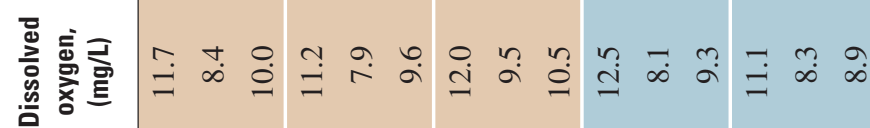

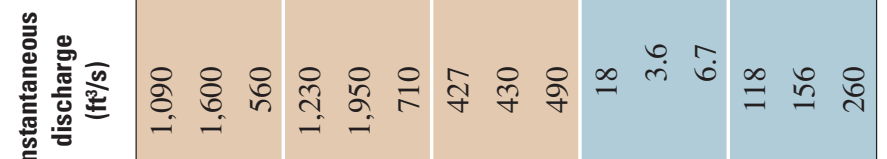

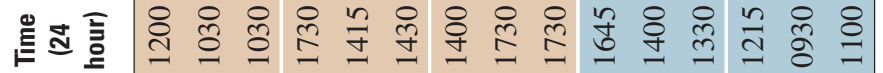

。 就 :

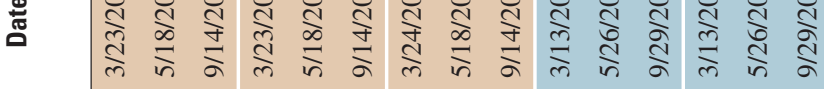

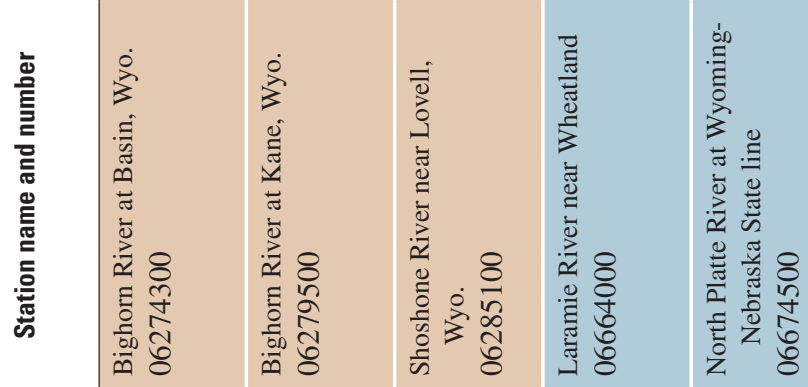

毫

密畐

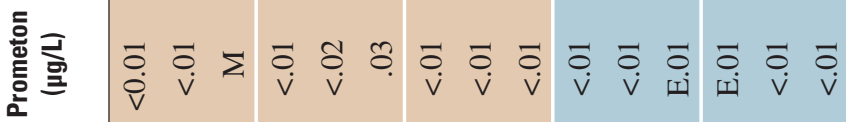

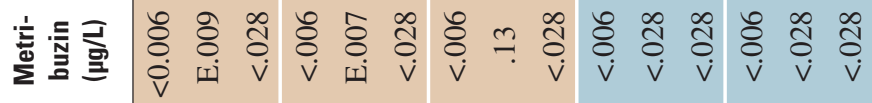

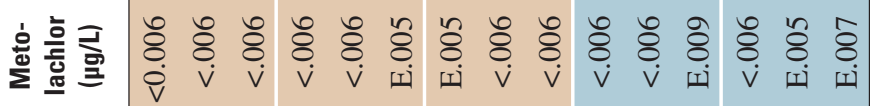

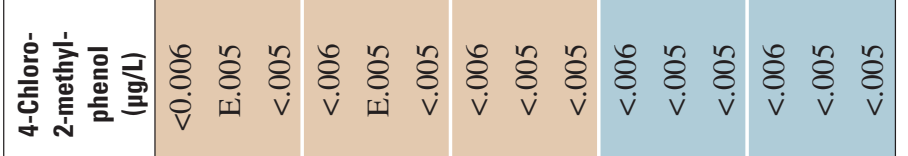

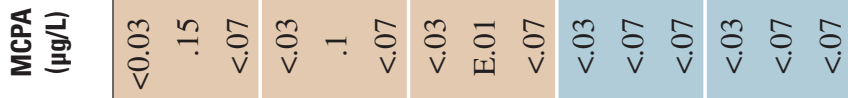

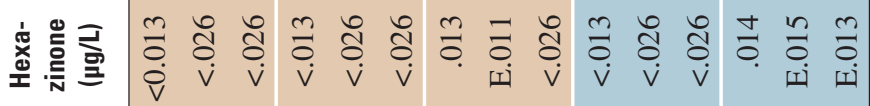

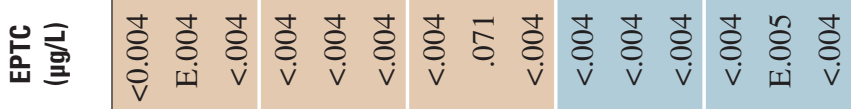

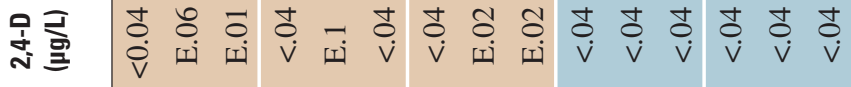

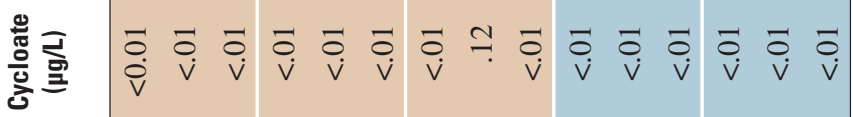

$\begin{array}{llllllllll} & & & & \\ 0\end{array}$

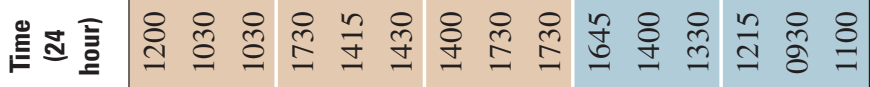

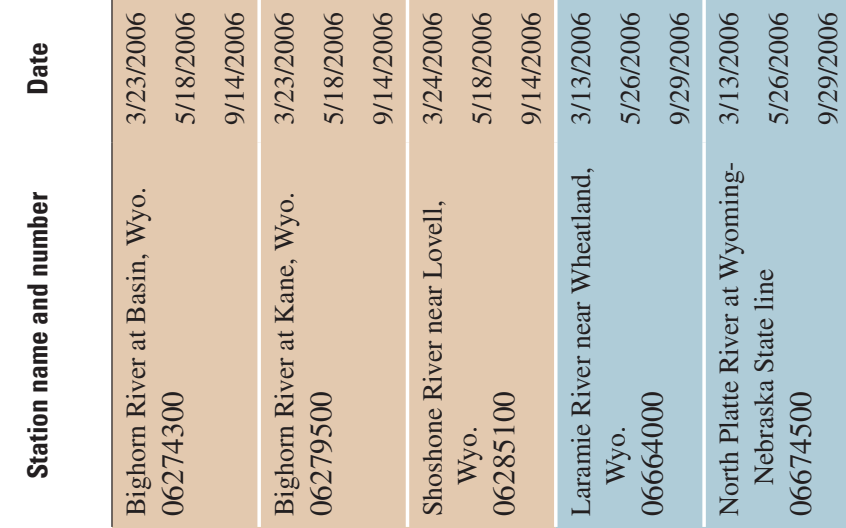



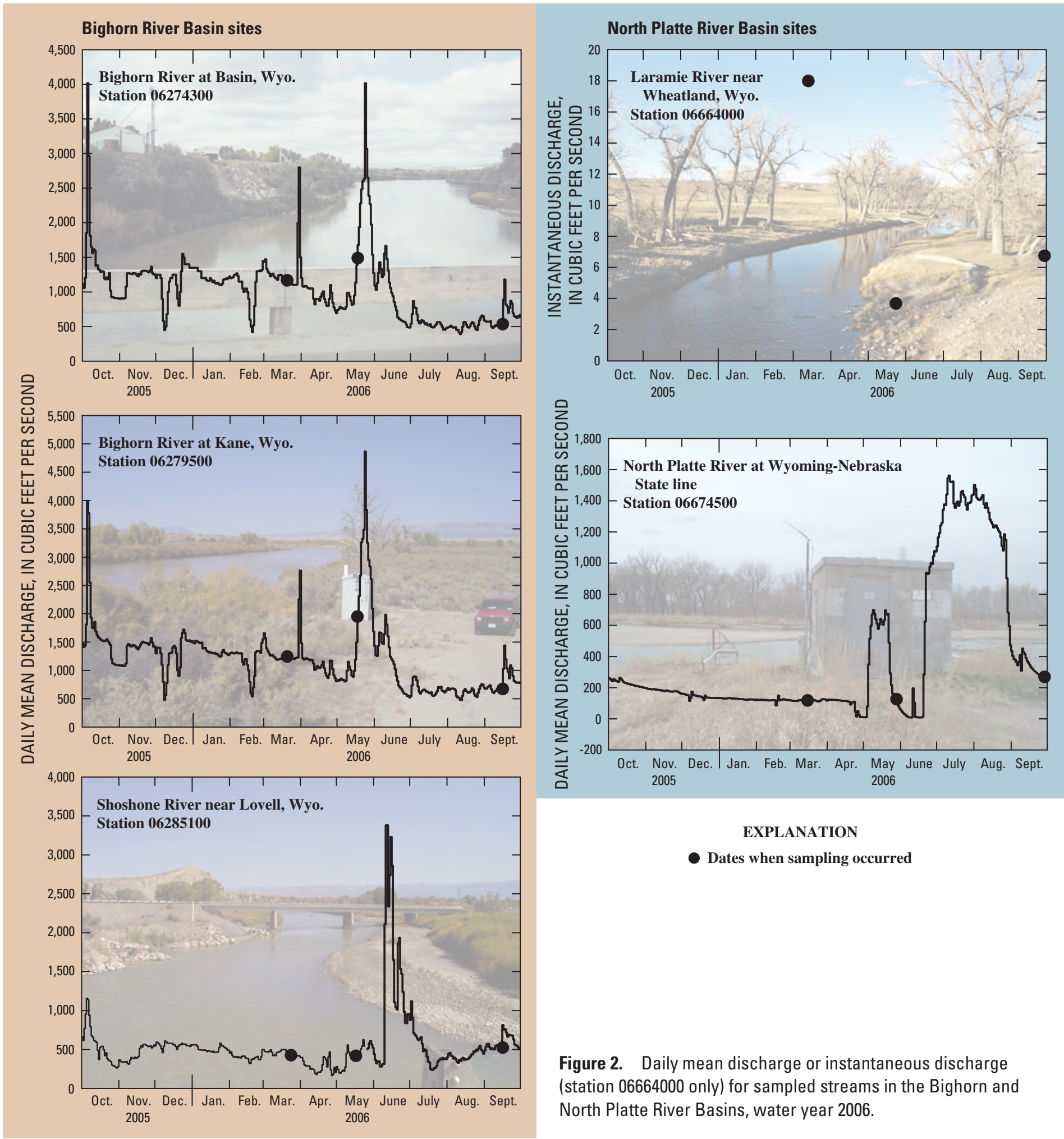

\section{EXPLANATION}

- Dates when sampling occurred

Figure 2. Daily mean discharge or instantaneous discharge (station 06664000 only) for sampled streams in the Bighorn and North Platte River Basins, water year 2006.

Solve and MCP), which was detected in a May sample at a concentration of $0.15 \mu \mathrm{g} / \mathrm{L}$ (micrograms per liter).

Eight different pesticides were detected in water samples collected in the NPRB. The most commonly detected pesticide was atrazine, detected in four of six samples collected in the NPRB. The pesticide with the largest concentration in the NPRB was the selective herbicide simazine (trade names Aquazine and Princep), which was detected in a September sample at a concentration of $0.08 \mu \mathrm{g} / \mathrm{L}$.

\section{References}

Furlong, E.T., Anderson, B.D., Werner, S.L., Soliven, P.P., Coffey, L.J., and Burkhardt, M.R., 2001, Methods of analysis by the U.S. Geological Survey National Water Quality Laboratory--Determination of pesticides in water by graphitized carbon-based solid-phase extraction and highperformance liquid chromatography/mass spectrometry: U.S. Geological Survey Water-Resources Investigations Report 01-4134, 73 p.

Meister, R.T., 2002, Farm Chemicals Handbook: Willoughby, Ohio, Meister Publishing Co., variable pagination. 
Table 2. Pesticides and degradation products analyzed for in samples.

[Pesticide trade names ${ }^{1}$ in parentheses if different from pesticide name; pesticides in color were detected in the corresponding basin: tan, Bighorn River Basin; blue, North Platte River Basin; dark red, both basins]

\begin{tabular}{|c|c|c|c|}
\hline Pesticide name & Pesticide name & Pesticide name & Pesticide name \\
\hline Acetochlor (Guardian, Harness, Relay) & Cyfluthrin (Decathlon) & & Nicosulfuron (Accent, OneHope) \\
\hline Acifluorfen (Blazer, Tackle 2S, Astic) & Cypermethrin & & Norflurazon (Zorial, Solicam) \\
\hline Alachlor (Alanex, Lasso, Shroud) & 2,4-D (Dacamine, Weed-B-Gon) & $\begin{array}{l}\text { Fenuron } \\
\text { Fipronil (Regent) }\end{array}$ & Oryzalin (Surflan) \\
\hline Aldicarb (Temik) & 2,4-D methyl ester & \multirow[b]{2}{*}{$\begin{array}{l}\text { Fipronil sulfide (Fipronil degradation } \\
\text { product) }\end{array}$} & Oxamyl (Vydate) \\
\hline $\begin{array}{l}\text { Aldicarb sulfone (Standak, Aldicarb degra- } \\
\text { dation product) }\end{array}$ & 2,4-DB (Butoxone, Butyrac) & & Oxyfluorfen (Offside, Goal) \\
\hline $\begin{array}{l}\text { Aldicarb sulfoxide (Aldicarb degradation } \\
\text { product) }\end{array}$ & Dacthal monoacid (Dacthal degradation & \multirow{2}{*}{$\begin{array}{l}\text { Fipronil sulfone (Fipronil degradation } \\
\text { product) } \\
\text { Flumetsulam (Broadstrike) }\end{array}$} & \multirow{2}{*}{$\begin{array}{l}\text { Paraoxon-methyl (Parathion-methyl degra- } \\
\quad \text { dation product) } \\
\text { Parathion-methyl (Alkron, Bladan, Fighter) }\end{array}$} \\
\hline Atrazine (Aatrex, Atranex) & product) & & \\
\hline Azinphos-methyl (Guthion, Crysthyon) & $\begin{array}{l}\text { Desulfinylfipronil (Fipronil degradation } \\
\text { product) }\end{array}$ & $\begin{array}{l}\text { Fluometuron (Cotoran) } \\
\text { Fonofos (Dyfonate) }\end{array}$ & \multirow{2}{*}{$\begin{array}{l}\text { Pendimethalin (Prowl, Stomp) } \\
\text { cis-Permethrin (Ambush, Pounce) } \\
\text { Phorate (Thimet, Rampart) }\end{array}$} \\
\hline $\begin{array}{l}\text { Azinphos-methyl-oxon (Azinphos-methyl } \\
\text { degradation product) }\end{array}$ & $\begin{array}{l}\text { Desulfinylfipronil amide (Fipronil degrada- } \\
\text { tion product) }\end{array}$ & Hexazinone (Velpar) & \\
\hline Barban & Diazinon (Basudin, Spectracide, Knoxout) & $\begin{array}{l}\text { 3-Hydroxycarbofuran (Carbofuran degra- } \\
\text { dation product) }\end{array}$ & Phosmet (Imidan) \\
\hline Benfluralin (Balan, Benefin) & $\begin{array}{l}\text { 3,4-Dichloroaniline (Chloramben degrada- } \\
\text { tion product) }\end{array}$ & $\begin{array}{l}\text { 2-Hydroxy-4-isopropylamino-6-ethyl- } \\
\text { amino-s-triazine }\{\text { OIET }\} \text { (Atrazine } \\
\text { degradation product) }\end{array}$ & Prometon (Pramitol, Gesafram) \\
\hline Bensulfuron-methyl (Escuri, Londax) & $\begin{array}{l}\text { 3,5-Dichloroaniline (Diuron degradation } \\
\text { product) }\end{array}$ & \multirow{2}{*}{$\begin{array}{l}\text { Imazaquin (Scepter) } \\
\text { Imazethapyr (New Path, Pursuit) }\end{array}$} & Prometryn (Cotton-pro) \\
\hline Bentazon (Basagram, Bentzone) & Dichlorprop (Weedone, Polymone) & & Propargite (Comite, Omite) \\
\hline Bromacil (Hyvar X) & Dichlorvos (DDVP) & Imidacloprid (Admire, Provado) & Propham (Chem Hoe) \\
\hline Bromoxynil (Buctril, Brominal, Agristar) & Dicrotophos (Bidrin) & \multirow{5}{*}{$\begin{array}{l}\text { Isofenphos (Oftanol, Lighter) } \\
\text { lambda-Cyhalothrin (Charge, Karate) } \\
\text { Linuron (Linurex, Lorox) } \\
\text { Malaoxon (Malathion degradation product) } \\
\text { Malathion (Cythion, Malaspray) }\end{array}$} & Propiconazole (Banner) \\
\hline Carbaryl (Carbatox, Sevin) & Dieldrin (Panoram D-31, Octalox) & & cis-Propiconazole (Banner) \\
\hline Carbofuran (Furadan, Futura) & $\begin{array}{l}\text { 2,6-Diethylaniline (Alachlor degradation } \\
\text { product) }\end{array}$ & & trans-Propiconazole (Banner) \\
\hline Chloramben, methyl ester (Chloramben) & Dimethoate (Trounce) & & Propoxur (Baygone, Suncide) \\
\hline $\begin{array}{l}\text { Chlordiamino-s-triazine }\{\mathrm{CAAT}\} \text { (Atrazine } \\
\text { degradation product) }\end{array}$ & Dinoseb (Premerge) & & Propyzamide (Kerb) \\
\hline Chlorimuron-ethyl (Classic, Darban, Lory) & Diphenamid (Dymid, Enide) & MCPA (Solve, MCP) & Simazine (Aquazine, Princep) \\
\hline $\begin{array}{l}\text { 2-Chloro-2,6-diethylacetanilide (Alachlor } \\
\text { degradation product) }\end{array}$ & Disulfoton (Di-Syston) & MCPB (Butoxone M40, Thistrol) & Sulfometuron-methyl (Oust) \\
\hline $\begin{array}{l}\text { 2-Chloro-4-isopropylamino-6-amino-s- } \\
\text { triazine }\{\text { CIAT\} (Atrazine degrada- } \\
\text { tion product) }\end{array}$ & $\begin{array}{l}\text { product) } \\
\text { Diuron (Durashield, Karmex) }\end{array}$ & $\begin{array}{l}\text { Metalaxyl (Apron, Ridamil, Subdue) } \\
\text { Methidathion (Supra) }\end{array}$ & Tebuconazole (Elite) \\
\hline $\begin{array}{l}\text { 2-Chloro-6-ethylamino-4-amino-s-triazine } \\
\text { \{CEAT }\} \text { (Atrazine degradation product) }\end{array}$ & $\begin{array}{l}\text { alpha-Endosulfan (Thiodan, Tiovel) } \\
\text { Endosulfan sulfate (alpha-Endosulfan }\end{array}$ & Methomyl (Lannate, Nudrin) & Tebuthiuron (Graslan, Spike) \\
\hline $\begin{array}{l}\text { 4-Chloro-2-methylphenol (MCPA degrada- } \\
\text { tion product) }\end{array}$ & & Metolachlor (Bicep, Dual) & Terbacil (Sinbar, Herbicide 732) \\
\hline $\begin{array}{l}\text { 3(4-Chlorophenyl)-1-methyl urea (Diuron } \\
\text { degradation product) }\end{array}$ & Ethion (Ethanox, Rhodocide) & Metsulfuron methyl (Ally, Escort) & Terbufos (Counter, Contraven) \\
\hline Chlorpyrifos (Dursban, Lorsban) & Ethoprophos (Mocap, Prophos) & Molinate (Hydram, Ordram) & \\
\hline Clopyralid (Stinger, Lontrel) & $\begin{array}{l}\text { 2-Ethyl-6-methylaniline (Metalachlor } \\
\text { degradation product) }\end{array}$ & Myclobutanil (Eagle, Nova) & Tribufos (DEF 6) \\
\hline Cyanazine (Bladex) & Fenamiphos & 1-Naphthol (Carbaryl degradation product) & Triclopyr (Garlon) \\
\hline Cycloate (Ro-Neet) & $\begin{array}{l}\text { Fenamiphos sulfone (Fenamiphos degrada- } \\
\text { tion product) }\end{array}$ & Neburon (Granurex, Propuron) & Trifluralin (Treflan, Trim) \\
\hline
\end{tabular}

${ }^{1}$ The use of trade, product, industry, or firm names is for descriptive purposes only and does not imply endorsement by the U.S. Government or the Wyoming State Government.

Sandstrom, M.W., Stroppel, M.E., Foreman, W.T., and Schroeder, M.P., 2001, Methods of analysis by the U.S. Geological Survey National Water Quality Laboratory-Determination of moderate-use pesticides and selected degradates in water by $\mathrm{C}-18$ solid-phase extraction and gas chromatography/mass spectrometry: U.S. Geological Survey WaterResources Investigations Report 01-4098, 70 p.

U.S. Geological Survey, 1997 to 2004, National field manual for the collection of water-quality data: U.S. Geological Survey Techniques of Water-Resources Investigations, book 9, chaps. A1-A9, 2 v., variously paged. [Also available online at http://pubs.water.usgs.gov/twri9A. Chapters originally were published from 1997-1999; updates and revisions are ongoing and are summarized at: http://water.usgs.gov/owq/ FieldManual/mastererrata.html]

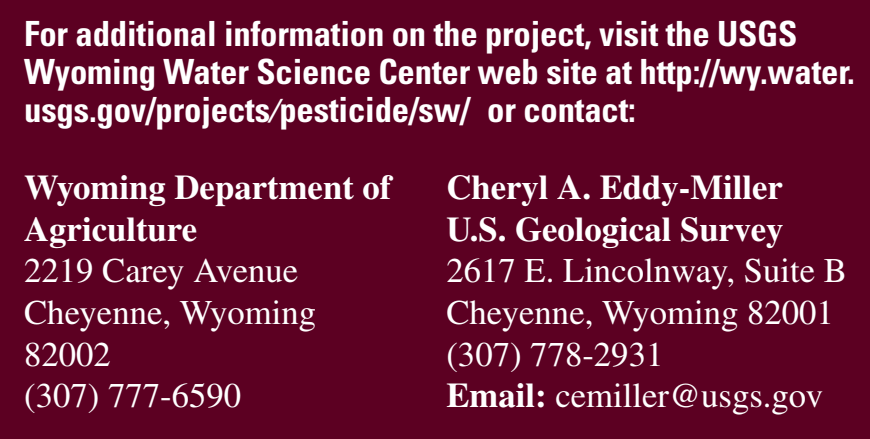

